\title{
Complete Internal Carotid Artery Occlusion Presenting as "Seizures"
}

\author{
Shah-Naz Hayat Khan
}

\begin{abstract}
Limb shaking is a rare and atypical presentation for a transient ischemic attack. It can be described as a "cerebral ischemic claudication" due to attenuation in an already compromised cerebral perfusion. The uncharacteristic presentation may lead to confusion and misdiagnosis, for example, focal epilepsy, delaying appropriate therapy. We describe such a case highlighting the clinical implications and options for addressing the underlying cerebrovascular pathology. A 57-years-old female with a 2 weeks history of slurred speech and involuntary shaking of the right upper and lower extremities lasting for a few minutes, which was not associated with loss of consciousness or amourosis fugax. Neuroimaging demonstrated a complete left internal carotid artery occlusion at its origin and a severe focal stenosis of the proximal external carotid artery. Supraclinoidal internal carotid artery was reconstituted via collaterals between ophthalmic artery and branches of left internal maxillary artery. The right internal carotid contributed via the anterior communicating artery. An external carotid endarterectomy was performed, resulting in a complete resolution of her symptoms. In conclusion, limb shaking as a manifestation of carotid occlusion is a rare phenomenon. To prevent misdiagnosis and appropriate treatment, cerebral ischemic claudication must always be considered in the appropriate setup. Any treatment must preserve the collaterals supplying the compromised cerebral vasculature.
\end{abstract}

Keywords: Transient ischemic attack; Limb shaking; Carotid artery occlusion; EC-IC bypass; Carotid endarterectomy; Carotid angioplasty and stenting

\section{Introduction}

Limb shaking is a rare manifestation of a transient ischemic attack (TIA) [1]. The atypical presentation in a patient with

Manuscript accepted for publication December 14, 2012

Saint Francis Spine and Neurosurgery Center, Saint Francis Hospital, 2122 Manchester Expressway, Columbus GA 31904, USA.

Email: khan@sasktel.net

doi: http://dx.doi.org/10.4021/jnr161w carotid occlusive disease may be mistaken for focal epilepsy, resulting in confusion or delayed diagnosis [1]. It is believed to occur because of the low perfusion of the brain [2]. We describe the presentation, neuroimaging and treatment of such a case.

\section{Case Report}

A 57-years-old obese, diabetic, hypertensive, hyperlipidemic, heavy smoking, Caucasian female was transferred to our institution with diagnosis of recurrent TIAs. The patient complained of "seizures" commencing 2 weeks prior to presentation. These comprised of involuntary coarse shaking and complaints of weakness of right arm and leg, along with slurred speech lasting approximately 5 minutes. The symptoms occurred several times a day with very mild exertion, for example, standing up from a lying position. There was no associated loss of consciousness. The patient denied any history of amourosis fugax. Her physical examination was unremarkable, including normal motor strength. Electroencephalography did not indicate seizure activity and demonstrated bitemporal delta wave activity, consistent with

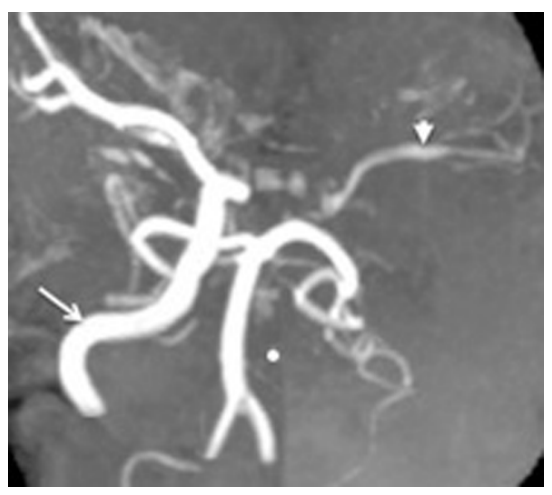

Figure 1. MRA demonstrating the absent Left ICA. The $(\bullet)$ highlights the site where the counterpart to the readily appreciated right ICA (arrow) should have been. The left MCA and its branches are visible (arrowhead), indicative of their reconstitution. The posterior circulation comprising of the two vertebral arteries confluencing into the basilar artery and their branches are also seen. 

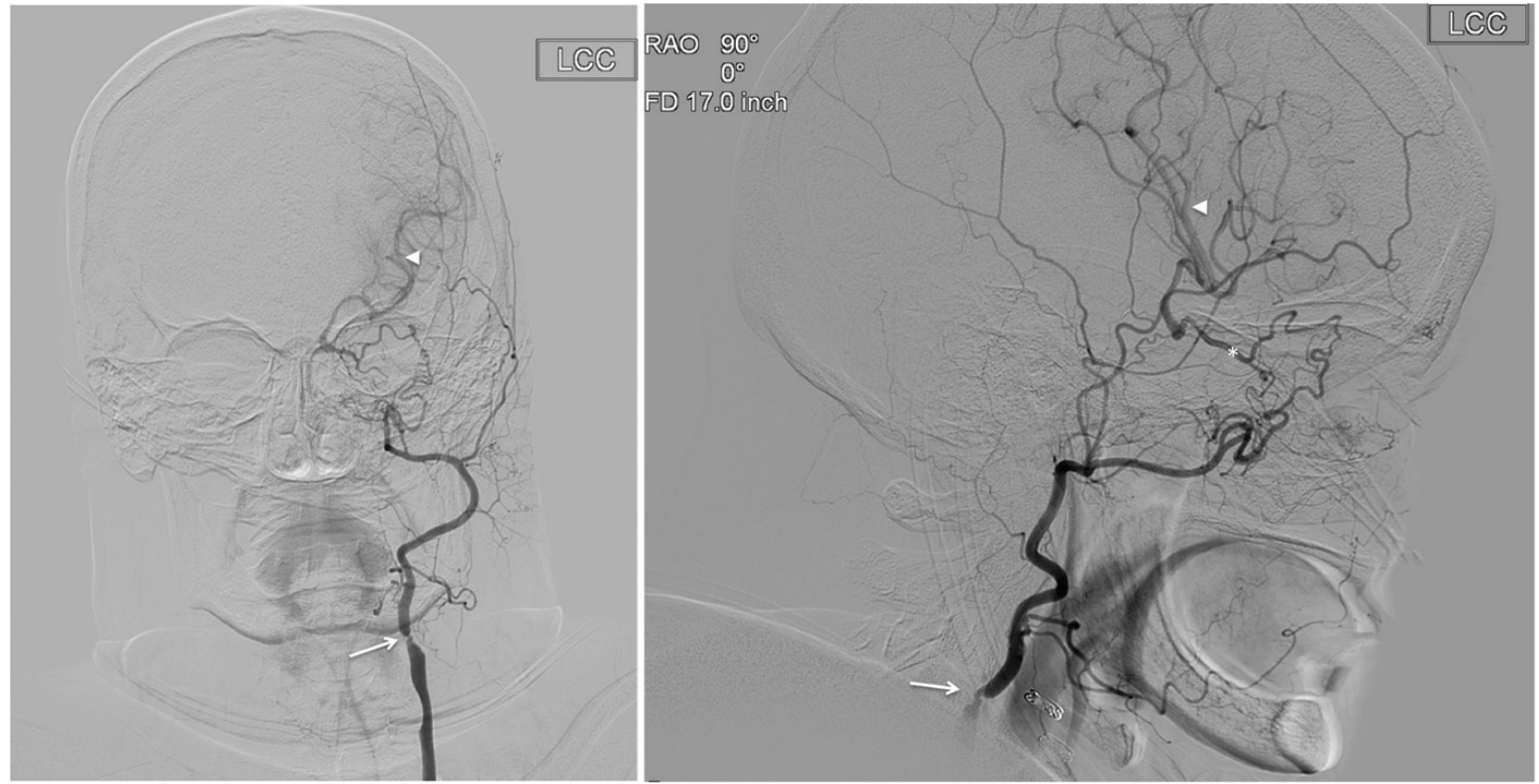

Figure 2. Catheter angiography of left common carotid artery in (a) anteroposterior and (b) lateral projections demonstrates the absence of left ICA. The ECA and its branches are appreciated. There is a severe stenosis of proximal ECA (arrow). The ethmoidal branches of the internal maxillary artery are anastomosing with the ethmoidal branches of the ophthalmic artery $\left({ }^{*}\right)$. The retrograde flow from the ECA via the opththalmic artery results in the reconstitution of the left MCA (arrowhead).

cerebrovascular disease. Magnetic resonance angiography (MRA) (Fig. 1) and catheter angiography (Fig. 2) demonstrated complete left internal carotid artery (ICA) occlusion at its origin. The patient's left middle cerebral artery (MCA) was supplied by a reconstitution of supraclinoidal ICA via collaterals between ophthalmic artery and branches of left internal maxillary artery. The left anterior cerebral artery (ACA) was supplied by the right ICA via the anterior communicating artery (Fig. 3). A severe focal stenosis was noted in the proximal external carotid artery (ECA) (Fig. 2), which was addressed by endarterectomy. There was a complete resolution of her symptoms post operatively.

\section{Discussion}

Limb shaking, also called, cerebral ischemic claudication, is believed to occur because of the low perfusion of the brain [2]. Symptoms typically occur upon sitting up or standing with a resultant "minor" compromise of cerebral perfusion, with rapid resolution on lying down.

The collateral blood supply is vital in such patients. When there is ipsilateral ICA occlusion, collateral flow to the MCA can occur from the contralateral ICA via the anterior communicating artery, the vertebrobasilar circulation via posterior communicating artery, or leptomeningeal anastomosis (LMA) from the above mentioned areteries [1,3]. The ECA can also supply collaterals from its maxillary or facial branches via ophthalmic artery, from the pterygopalatine network formed by the distal maxillary branches or, the oropharyngeal network formed by the ascending pharyngeal artery. The latter two networks may communicate with the petrous

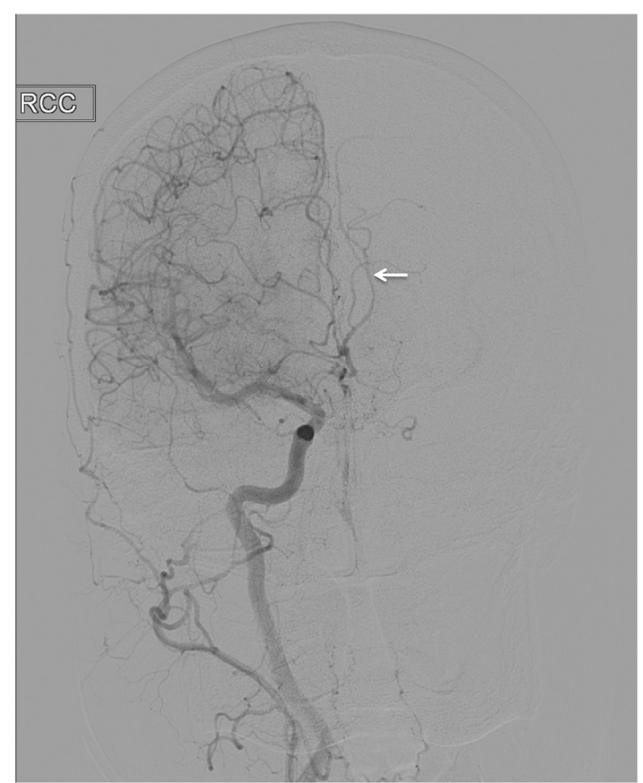

Figure 3. Catheter angiography of right ICA demonstrating a hypoplastic right ACA which also supplies the left ACA Territory (arrow). The left ACA branches were not visualized on contralateral (left) injection. 
ICA via the vidian artery $[4,5]$. Branches of the Maxillary artery and ophthalmic artery may also provide collateral flow to the ICA via the inferolateral trunk which arises from the cavernous ICA. The ophthalmic artery is the most common collateral between ICA and ECA in patients with ICA Occlusion [4]. Patients with ICA occlusion and limb shaking were found to be seven times more dependent on LMA than those without limb shaking.

Symptomatic ICA occlusions have been addressed by ECA-ICA bypass with good outcomes [1]. Our patient underwent an external carotid endarterectomy because she had well established collateral flow via the ophthalmic artery, the limiting factor being a severe stenosis in the proximal ECA. Another option would have been angioplasty with or without stenting. However, the patient was noted to have renal insufficiency, making endarterectomy a better option as endovascular intervention requires contrast administration. Additionally, in patients dependent upon leptomeningeal collaterals, serious thought should be given before undertaking cranial procedures that may risk disrupting the same.

\section{Conclusion}

This case highlights the importance of keeping in mind posture induced limb shaking as a manifestation of severe hemi- spheric ischemia due to ICA occlusion with collateral supply other than the circle of Willis, for example, opthalamic and leptomeningeal vessels. The treatment selected should maintain the integrity of the vital collateral circulation, upon which the patient is dependent.

\section{References}

1. Baquis GD, Pessin MS, Scott RM. Limb shaking--a carotid TIA. Stroke. 1985;16(3):444-448.

2. Tatemichi TK, Young WL, Prohovnik I, Gitelman DR, Correll JW, Mohr JP. Perfusion insufficiency in limb-shaking transient ischemic attacks. Stroke. 1990;21(2):341-347.

3. Brozici M, van der Zwan A, Hillen B. Anatomy and functionality of leptomeningeal anastomoses: a review. Stroke. 2003;34(11):2750-2762.

4. Takeuchi M, Kuwayama N, Kubo M, Umemura K, Hirashima Y, Endo S. Vidian artery as a collateral channel between the external and occluded internal carotid arteries--case report. Neurol Med Chir (Tokyo) 2005;45:470471.

5. Quisling RG, Rhoton AL, Jr. Intrapetrous carotid artery branches: radioanatomic analysis. Radiology. 1979;131(1):133-136. 